\title{
An Optimized Routing OLSR Protocol with Low Control Overhead for UAV Ad Hoc Networks
}

\author{
Qizheng Zhu*, Zhou Zhou, Jie Yang, Zeliang Fu \\ Key Laboratory of Mobile Communication Technology, Chongqing University of Posts and Telecommunications, Chongqing, China \\ Email address: \\ 826367512@qq.com (Qizheng Zhu),449314625@qq.com (Zhou Zhou),2302170498@qq.com (Jie Yang),935388623@qq.com (Zeliang Fu) \\ ${ }^{*}$ Corresponding author
}

To cite this article:

Qizheng Zhu, Zhou Zhou, Jie Yang, Zeliang Fu. An Optimized Routing OLSR Protocol with Low Control Overhead for UAV Ad Hoc Networks. American Journal of Networks and Communications. Vol. 10, No. 1, 2021, pp. 6-12. doi: 10.11648/j.ajnc.20211001.12

Received: April 19, 2021; Accepted: May 19, 2021; Published: May 24, 2021

\begin{abstract}
OLSR (Optimized Link State Routing) protocol is a priori Routing protocol applied in MANET network. All nodes in the network have Routing information to other nodes locally and need to send a large number of control messages to maintain the topology information of the whole network. In UAV application scenarios, with the increase of node density, the control overhead will be too high and the problems of hidden terminals will be intensified. Secondly, due to the fast moving speed of UAVs, when the neighboring UAVs have moved out of the communication range of the local node, the local node still retains the routing information of the neighbor node, leading to the problem of packet loss when the link information is updated behind time. Aiming at the above problems, an OLSR protocol with low control overhead and optimal routing is proposed. The protocol uses the incremental HELLO message mechanism to ensure that only incremental information is exchanged when the network topology changes slowly, and the control overhead is greatly reduced. When calculating routing, in the range of $\mathrm{n}$ hops, the more stable link is selected under the same number of hops. Simulation results show that the proposed algorithm can significantly improve the success rate of packet transmission and end-to-end delay, and reduce the system control overhead.
\end{abstract}

Keywords: OLSR Protocol, Control Overhead, Optimal Routing, UAV, The Channel Occupancy Rate

\section{Introduction}

With the development of military technology in the direction of unmanned and intelligent in recent years, some intelligent networks such as UAV Ad-Hoc Network (UANET) have received more and more attention from various countries $[1,2]$. In addition to the characteristics of traditional ad hoc network, such as no center, self-organization [3], multi hop routing [4], dynamic topology [5], etc. UAV ad hoc network has unique characteristics different from ad hoc network due to its fast mobile speed and complex communication environment [6-9]. At present, relevant scholars have conducted some research on the UAV ad hoc network protocol. Literature $[10,11]$ proposed a geographical location-based OLSR protocol, adding a location field to the HELLO message. Since additional content was added to the original HELLO packet, it brought some additional control overhead. In [12], a new MPR selection mechanism is proposed, which uses mobile stability as the MPR node selection criterion, thereby improving the stability of the protocol, but the new selection algorithm cannot guarantee that the selected MPR set is the smallest, Therefore, the number of TC messages sent and forwarded in the network may be increased, resulting in additional control overhead. In response to the problem that the single-hop coverage of the node is small, which leads to an increase in the number of routing hops, Alshbatat proposed an improved mechanism of using directional antenna to expand the communication coverage to reduce the number of hops in literature [13]. However, this mechanism requires to design a completely new MAC layer protocol to control the physical layer. The improved algorithm is difficult to implement and has no scalability and practical application. Due to the poor wireless channel quality in the UAV ad hoc network, it is easy to send a disconnection. In literature [14], signal strength was introduced as a criterion for link quality in this issue, and a link based on link quality and traffic was proposed. The load-aware routing protocol (LTA-OLSR), but in this paper, only the signal strength is considered when routing may lead to an increase in route hops, and there is no balanced choice between signal strength and shortest hops. 
After researching the existing literature, it is found that the channel utilization rate of the UAV ad hoc network in a large-scale application environment with dense nodes is too low and there are hidden terminal problems, which may cause the collision of the underlying data packets to increase; besides, The highly dynamic nature of the UAV ad hoc network may cause problems such as transmission link interruption. Therefore, based on the relevant research in [14], this paper proposes a low control overhead optimized routing OLSR protocol for UAV ad hoc networks (LCO-OLSR).

The paper structure of this paper is as follows. The introduction of section I describes the current research status and possible problems of the UAV ad hoc network and OLSR protocol; the principle of the OLSR protocol is summarized in section II. It also clarifies the problems in the application of the OLSR protocol in the UAV ad hoc networks. Section III proposes corresponding improvement mechanisms for the problems in section II, and section IV gives a detailed description and performance analysis of the improved parts; In section $\mathrm{V}$, the improved mechanism proposed in section IV is simulated and verified, which proves the superiority of the improved mechanism compared with the existing mechanism; section VI summarizes the full text.

\section{Problems with OLSR Protocol of UAV Ad Hoc Network}

Due to the very fast-moving speed, uncertain movement direction, and complicated communication environment of the UAV, the original OLSR protocol will encounter loss of control messages, low channel utilization, and unstable routing when applied in the UAV ad hoc network. And that will not meet the actual demands in the UAV ad hoc networks [15-17], so the following will analyze these problems in detail.

\subsection{Loss of Control Message in Node Intensive Scenarios}

Since the network topology of the OLSR protocol is constructed by periodically broadcasting HELLO and TC control messages, there are certain requirements for the reliability of network communication. However, in the wireless application environment such as UAV ad hoc networks, hidden terminal problems may exist. As the density of nodes in the network increases, because the broadcast control packet of the OLSR protocol does not have an ACK mechanism when it is sent, the success rate of receiving HELLO and TC control packets will be reduced by the influence of hidden terminals in dense node scenarios, which will lead to a decline in network communication performance.

\subsection{Low Channel Utilization Rate in Dense Node Scenarios}

When the OLSR protocol is used in the UAV ad hoc network, a large number of HELLO and TC control messages need to be sent to maintain the routing update during the protocol networking, but as the node density increases, the transmission of control messages will occupy a large number of channels Resources, which leads to a decrease in the available bandwidth of the network and a decrease in throughput. As shown in Figure 1, the simulation shows that with the increase of node density, the channel occupancy rate of control messages increases exponentially, which will seriously affect the communication performance of the network.

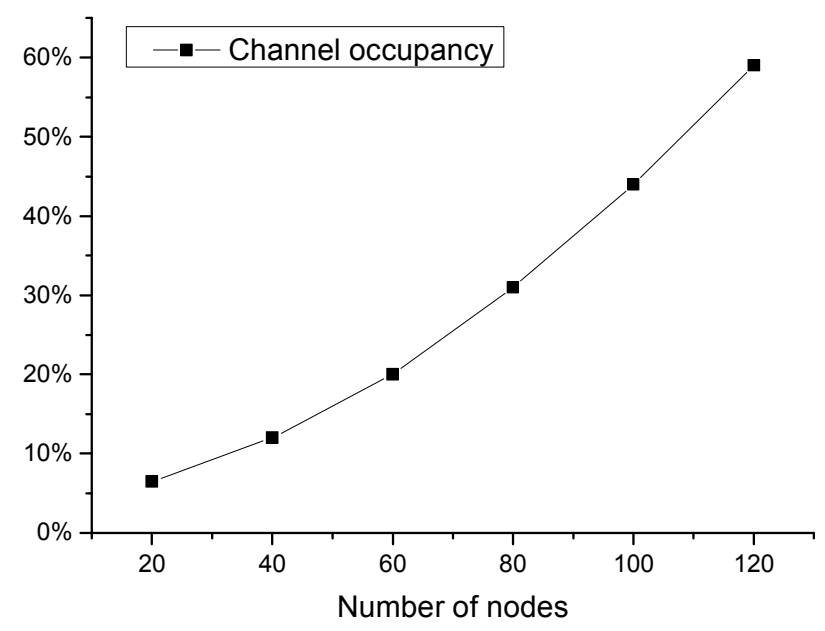

Figure 1. Relationship between channel occupancy and number of nodes.

\subsection{Routing Problem}

Because the classic OLSR protocol does not consider the impact of drone speed and communication distance on communication when calculating routes, and at the same time, UAVs often have multiple routes with the same hop count when communicating, as shown in Figure 2. At the same time, due to the fast-moving speed of the UAV, the symmetric link retention time defaults to $6 \mathrm{~S}$. During this period, the intermediate node in the routing path 2 may fly out of the communication range while data communication is in progress, resulting in the loss of data packets and seriously affecting network performance. However, the original OLSR routing protocol has no relevant selection criteria between route 1 and route 2 . Literature [14] only considers the signal strength of the link, but the link with higher signal strength may have a longer hop count, which leads to reduce link reliability and increase communication delay.

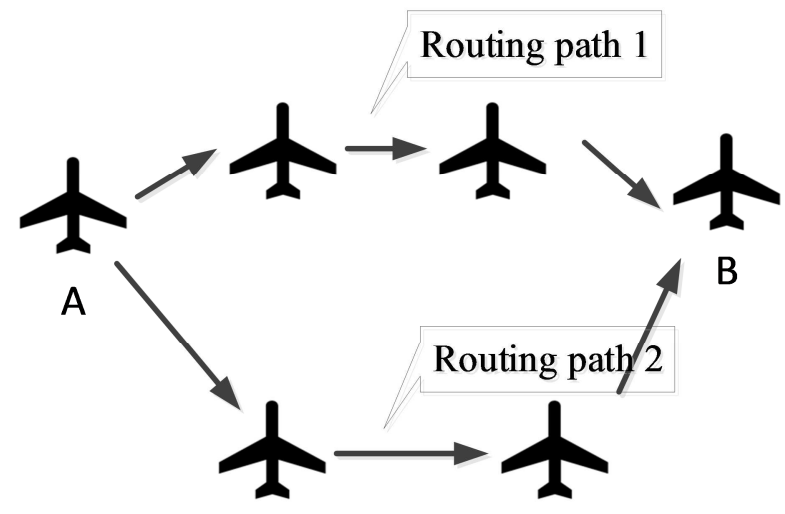

Figure 2. Routing status. 


\section{Improved LCO-OLSR Algorithm}

To solve the problems faced by the OLSR protocol of the UAV ad hoc network, this paper proposes an optimized routing OLSR protocol with low control overhead for the UAV ad hoc network, which performs the neighbor discovery process and routing computer system of the original protocol related improvements are made, the specific improvement plan is as follows.

\subsection{Incremental HELLO Message Sending Mechanism}

In the UAV application scenario, dense nodes and fast-moving speed lead to high channel occupancy rates, and there are hidden terminals, which lead to a low success rate of broadcast message reception and other problems. A mechanism for reducing the packet length of the HELLO message and reducing the total control overhead is proposed to improve network performance in scenarios such as high node density.

The new mechanism divides the sent HELLO messages into 3 types: HELLO keep messages (without carrying content), HELLO messages (with complete one-hop neighbor information), and HELLO incremental messages (with newly added one-hop node information). According to the type of HELLO message, the change of one-hop neighbors around a node can be divided into three categories: one-hop neighbors remain unchanged, one-hop neighbors are added, and one-hop neighbors are detached. The HELLO message sending strategies for the above three situations are discussed below.

1) One-hop neighbors remain unchanged: when the node topology changes slowly, one-hop neighbors do not change, and sending an empty packet HELLO message indicates that the one-hop neighbors have not changed.

2) Newly added one-hop neighbors: When a new node joins a one-hop neighbor, the normal neighbor-aware procedure is performed. During the neighbor-aware process, the newly joined node will obtain the complete one-hop neighbor information through the normal HELLO message. After the neighbor sensing is completed, the incremental information HELLO message is sent in the next three HELLO messages to inform the one-hop neighbor of the newly added HELLO message.

3) One-hop neighbor detachment: The three HELLO messages sent afterward are normal and contain complete one-hop neighbor information.

Regarding the mechanism, the one-hop neighbor changes and continuously sends HELLO messages for 3 cycles; if the neighbor node fails to receive the HELLO message for 3 consecutive cycles, the neighbor node will remove the information of the node locally and enter the neighbor sensing process again. This mechanism ensures that when the network topology changes slowly, only incremental information needs to be exchanged, which greatly reduces HELLO control overhead.

\subsection{Optimized Routing Computer System}

In literature [14], the signal strength is proposed as an optimization condition for routing, but there is no comprehensive consideration between the signal strength and the shortest hop count. Therefore, based on the literature [14], when there are multiple routes with the same hop count, the route calculation algorithm is further optimized.

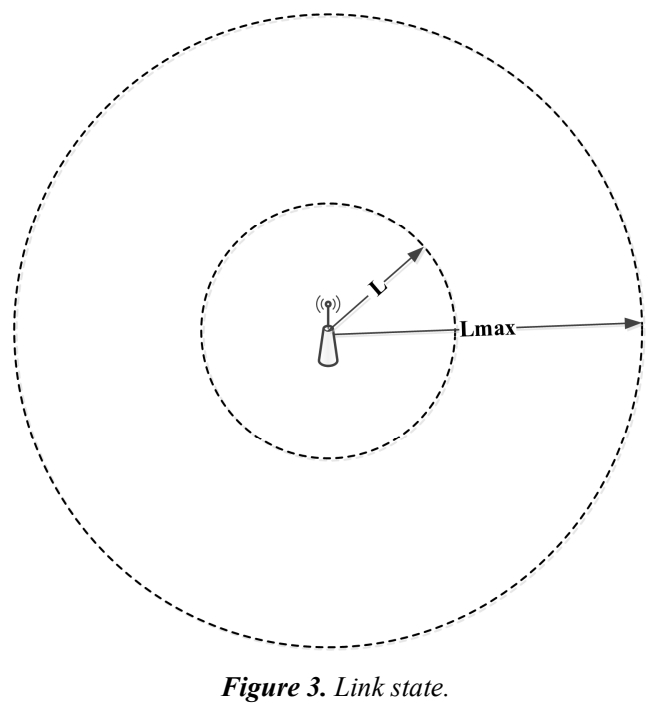

According to the degree of link stability between adjacent nodes, the single-hop link of a node can be divided into a stable link and an ordinary link. As shown in Figure 3, the maximum communication distance of the node is $\mathrm{L}_{\max }$, the maximum moving speed of the drone is $\mathrm{V}_{\max }$, and the single communication time is t. We define a stable link as a stable link, where a node cannot fly out of the communication range within time $t$ and keeps communication uninterrupted. Then, the communication range threshold of a single-hop stable link is shown in equation (1), and links that are not within this threshold are called ordinary links.

$$
L_{t h r}=L_{\text {max }}-V_{\text {max }} * t
$$

When the OLSR protocol selects the next-hop node, the node must be a symmetric node. Since the symmetric node retention time is $6 \mathrm{~s}$, in order to avoid the interruption of the single hop link during the symmetric node retention time $\mathrm{t}=6 \mathrm{~s}$, in the divided link-state The threshold is given as:

$$
L_{\text {thr }}=L_{\max }-6 * V_{\max }
$$

When the UAV node communicates with the surrounding UAVs, due to the transmitter's transmit power, antenna gain and wireless transmission-related losses mainly depend on the hardware equipment and communication environment, its value is often a fixed value and cannot be changed. Then the received power of the receiver is only related to the communication distance $\mathrm{d}$, as shown in (3).

$$
P_{r}(d)=\frac{G_{t} G_{r} P_{t} \lambda^{2}}{(4 \pi)^{2} d^{2} L}
$$

In the above formula, $G_{t}$ is the wireless gain of the transmitter, $G_{r}$ is the wireless gain of the receiver, $P_{t}$ is the transmission power of the transmitter, $\lambda$ is the electromagnetic wave wavelength, $\mathrm{d}$ is the communication distance, and $\mathrm{L}$ is 
the loss related to wireless transmission. When the communication distance is the single-hop stable link threshold, the optimal received power threshold of the receiver's single-hop stable link can be obtained as shown in equation (4).

$$
P_{r}\left(L_{t h r}\right)=\frac{G_{t} G_{r} P_{t} \lambda^{2}}{(4 \pi)^{2} L_{t h r}{ }^{2} L}=\frac{G_{t} G_{r} P_{t} \lambda^{2}}{(4 \pi)^{2}\left(R-6 * V_{\max }\right)^{2} L}
$$

When the UAV node receives the data packets of the surrounding nodes, the stability of the link related to the sending node can be judged by counting the received power $P$. When the relationship of the received power is $\mathrm{P}>\mathrm{P}_{\mathrm{thr}}$, the sending node is listed as a stable node, and the link communicated with the node is marked as a stable link; otherwise, it is marked as a normal link.

Since receiving data packets between neighboring nodes can only know the link status between one-hop neighbors, it is necessary to broadcast the link status between neighboring nodes to the entire network. When sending a TC message, use the Reserved field in the TC message packet structure to carry the link state information, indicating that the number of nodes represented by the previous Link_Stable_Num is a communication link stable node. The modified TC message packet format is shown in Figure 4.

\begin{tabular}{|c|c|}
\hline 0 & 16 \\
\hline ANSN & Link_State_Num \\
\hline Stable MPR Select or Address \\
\hline Stable MPR Selector Address \\
etc \\
\hline Normal MPR Selector Address \\
\hline Noamal MPR Selector Address \\
etc \\
\hline
\end{tabular}

Figure 4. Improved TC message packet structure.

When the node receives the TC message, it adds the previous address indicated by Link_Stable_Num to the topology table and marks the corresponding entry as a stable link; when the subsequent node is added to the topology table, it is marked as a normal link. The modified topology table is shown in the following table, in which the Link_State field is added to mark the link state between dest_addr and last_addr, which is 1 when it is stable link and is 0 when it is normal link.

\begin{tabular}{|l|l|l|l|l|}
\hline dest_addr & last_addr & seq_num & v_time & link_state \\
\hline
\end{tabular}

Figure 5. Improved topology table format.

The route calculation of LCO-OLSR protocol uses D algorithm, and on the premise of the shortest hops, choose the link that contains as many stable nodes as possible. Assume that the weight of a single-hop stable link is p1, and the weight of a single-hop ordinary link is $\mathrm{p} 2$, where $\mathrm{p} 2>\mathrm{p} 1$. The routing calculation process of the LCO-OLSR algorithm is as follows:

Step 1: Delete the content in the local routing table.

Step 2: One-hop symmetric neighbors are added as routing entries in sequence, and the communication distance is marked as $n=1$ and the multi-hop link weight is $m$. The destination address and the next-hop address are both neighbor node addresses.

Step 3: In the routing table, find the destination node $\mathrm{S}$ with the smallest number of communication hops, the multi-hop link weight and the smallest, and in turn find out whether other nodes can be reached through the node $\mathrm{S}$. If possible, create a new routing table entry. The destination node in the table entry is set to the current node, the next hop address is set to the address of node $\mathrm{S}$, the hop number is increased by 1 , and the multi-hop link weight value is updated to the previous hop weight value $m$ plus the current link Weight.

Step 4: Repeat Step 3 until all nodes are traversed, and no new nodes are added to the routing table entry. After the routing table is established, regular maintenance needs to be performed to check whether the routing table has expired. When the local symmetric neighbor or topology table changes, the route needs to be recalculated to maintain the timeliness of routing information.

When calculating the route, within $\mathrm{n}$ hops, when a more stable link is selected under the same hop count, $\mathrm{p} 1$ and $\mathrm{p} 2$ has a constraint relationship as $\left(n \mathrm{p}_{2}-n \mathrm{p}_{1}\right)<1$. Make the difference between the minimum total weight and the maximum total weight of the n-hop link not more than 1 to avoid the selection of a link with better link quality when routing in [14], which leads to a longer communication hop count Case.

\subsection{Performance Analysis of LCO-OLSR Algorithm}

In an ad hoc network, due to the dynamic changes of the network topology and the limitations of node energy, the factors that affect the network performance indicators are: the total number of nodes in the network, the node's packet sending rate, the size of the packet, the node's moving speed and the Link stability, etc. Aiming at the application scenarios of OLSR protocol applied to UAV ad hoc networks, the LCO-OLSR protocol proposes to reduce the redundant control overhead caused by the periodic broadcast of HELLO messages and optimize route calculation, improve the control overhead of OLSR protocol, and the success rate of packet sending. The performance in terms of throughput and latency is specifically analyzed as follows.

\subsubsection{Network Control Overhead Analysis}

In the OLSR routing protocol, routing information is mainly updated and maintained by nodes periodically interacting with neighbor maintenance messages (HELLO) and topology maintenance messages (TC), so the control overhead in the network can be calculated as follows.

When the ad hoc network is running, the length of the HELLO message generated by a certain node I in the unit time in the unit time is as follows: 


$$
\operatorname{Len}_{\text {hello }}=S Z_{\text {hello }} * \frac{1}{T_{\text {hello }}}
$$

Where $\mathrm{SZ}_{\text {hello }}$ is the average length of a single HELLO message, $T_{\text {hello }}$ is the sending period of a single HELLO message. Similarly, the length of the TC message that can be generated by the MPR node $\mathrm{j}$ per unit time is:

$$
L e n_{S_{-} T C}=S Z_{T C} * \frac{1}{T_{T C}}
$$

Where $\mathrm{SZ}_{\mathrm{TC}}$ is the length of a single TC message, and $\mathrm{N}_{\mathrm{m}}$ is the transmission period of the TC message. Considering

$$
\text { Total }=\sum_{i=1}^{N} \text { Len }_{\text {hello }}+\sum_{j=1}^{N_{M S}}\left(\operatorname{Len}_{S_{-} T C}+\operatorname{Len}_{T_{-} T C}\right) \quad=\sum_{i=1}^{N} S Z_{\text {hello }} * \frac{1}{T_{\text {hello }}}+\sum_{j=1}^{N_{M S}}\left(1+\left(N_{M S}-1\right) * N_{m}\right) * S Z_{T C} * \frac{1}{T_{T C}}
$$

According to (8), reducing the length of HELLO and TC messages or reducing the number of MPR nodes in the network can reduce the control overhead of the OLSR protocol and reduce the occupation of network bandwidth. The LCO-OLSR algorithm proposed in this paper uses an incremental HELLO message mechanism, which reduces the average length of HELLO message packets, which can effectively reduce the control overhead of the protocol.

\subsubsection{Success Rate and Delay Analysis}

We define the packet sending success rate as $\mathrm{S}$, the link quality as Q, and the single-hop link reliability as q, then the $\mathrm{n}$-hop link quality is as shown in the following formula, where $\mathrm{q}_{\mathrm{i}} \leq 1$.

$$
Q=\prod_{i=1}^{n} q_{i}
$$

It can be obtained from the above formula that when the number of communication hops increases, the quality of the n-hop link will deteriorate, and the success rate of packet sending will also decrease accordingly. Since LCO-OLSR protocol has added routing optimization selection on the basis of literature [14], selecting reliable links in N-hop links, so you can know the following.

$$
S_{L C O \_O L S R}>S_{O L S R}
$$

On the other hand, we define the total delay as D and $\mathrm{d}$ as the single-hop packet sending delay, then the n-hop link packet sending delay is as shown in the following formula.

$$
D=\sum_{i=1}^{n} d_{i}
$$

multi-point relay, the same TC message needs to be forwarded to its MPR neighbor nodes, so the total length of the TC message that MPR node $\mathrm{j}$ needs to forward in a unit time is:

$$
L e n_{T_{-} T C}=\left(N_{M S}-1\right) * N_{m} * S Z_{T C} * \frac{1}{T_{T C}}
$$

Where $\mathrm{N}_{M S}$ is the number of MPR nodes, and $\mathrm{N}_{m}$ is the average number of MPR nodes selected for each node. Because only MPR nodes generate and forward TC messages, the total routing overhead generated by the entire ad hoc network per unit time is:

In the paper, the MAC layer uses the CSMA/CA protocol, which is a competition-type access protocol. The CAMA/CA protocol requires a contention channel when sending data, and indicates the transmission duration. Because the LCO-OLSR protocol reduces the control overhead, reduces the channel load, and reduces the transmission time, thereby reducing the single-hop packet transmission delay $\mathrm{d}$, and reducing the total delay $\mathrm{D}$.

\subsection{Simulation Experiment Verification}

Select standard OLSR protocol, LCO-OLSR protocol and LTA-OLSR protocol as the comparison objects, and analyze and compare their important performance indicators such as success rate of packet sending, transmission delay, network control overhead through simulation experiments.

\subsubsection{Simulation Parameter Settings}

This article uses OPNET14.5 simulation software on the Windows XP platform to simulate the standard OLSR protocol, LTA-OLSR protocol and LCO-OLSR protocol. A total of 5 simulation scenarios are set in the article, respectively $20,40,60,80,100$ The nodes are evenly distributed in a rectangular area of $1500 \mathrm{~m}^{*} 1500 \mathrm{~m}$. The maximum moving speed of the drone is set to $10 \mathrm{~m} / \mathrm{s}$ and the maximum communication distance is $200 \mathrm{~m}$, then the single-hop link-state threshold is $\mathrm{L}_{\text {thr }}=140 \mathrm{~m}$ divided. The simulation parameters are shown in Table 1. The MAC layer uses the IEEE802.11 standard. Do 5 experiments for each scene and take the average of the experiment results.

Table 1. Simulation parameter settings.

\begin{tabular}{lllll}
\hline Number of nodes & Number of business flows (articles) & Package size (B) & Simulation time (s) & Simulation times (times) \\
\hline 20 & 3 & 1024 & 500 & 5 \\
40 & 3 & 1024 & 500 & 5 \\
60 & 3 & 1024 & 500 & 5 \\
80 & 3 & 1024 & 500 & 5 \\
100 & 3 & 1024 & 500 & 5 \\
\hline
\end{tabular}

\subsubsection{Analysis of Simulation Results}

1) Network control overhead analysis

Figure 5 shows that the LCO-OLSR protocol is superior to the standard OLSR protocol and LTA-OLSR protocol in terms of control overhead. Sending new HELLO messages and empty packet HELLO messages can significantly reduce control overhead and improve network performance. 


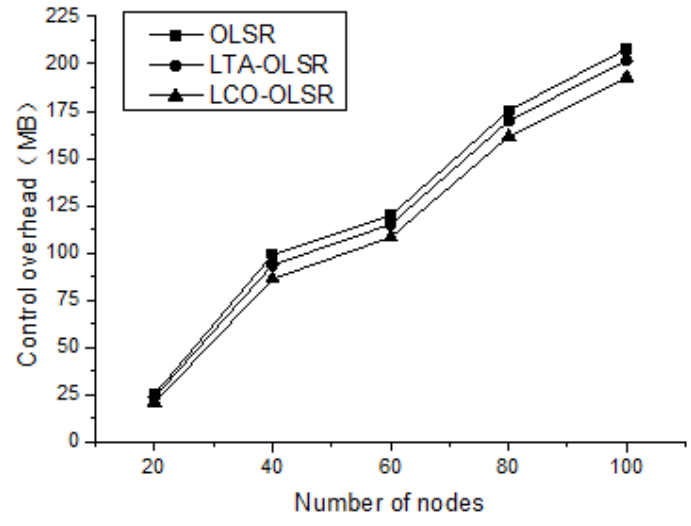

Figure 6. Control cost comparison.

2) Transmission delay analysis

Figure 6 shows that the LCO-OLSR protocol is superior to the OLSR protocol and the LTA-OLSR protocol in delay performance. Because the LCO-OLSR protocol reduces the control overhead through the incremental HELLO message mechanism, reduces the channel load, reduces the packet transmission delay, and reduces the total transmission delay. Besides, when the number of hops is equal, the LCO-OLSR protocol selects a more stable link, reducing the probability of erroneous retransmission of data packets and reducing the total delay. The LTA-OLSR protocol has poor latency performance in scenarios with 80 and 100 nodes. The reason is that as the number of communication hops increases, the link selection is not the shortest hop, which increases the total delay.

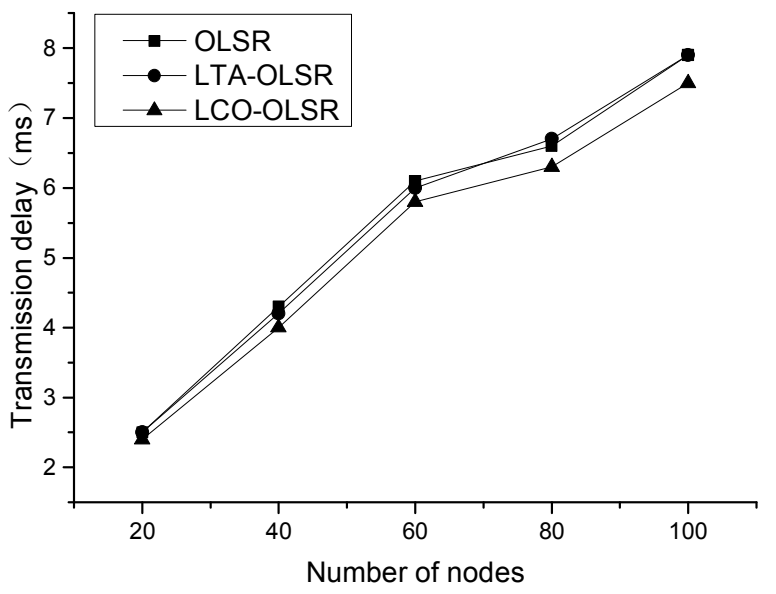

Figure 7. Comparison of transmission delay.

3) Analysis of success rate of outsourcing

Figure 7 shows that the LCO-OLSR protocol is superior to the OLSR protocol and LTA-OLSR protocol in the success rate index of packet sending. Among them, the LTA-OLSR protocol performs poorly in the scenario of 100 nodes, mainly because the number of communication hops will increase as the number of communication nodes increases. Because the LTA-OLSR protocol selects the next-hop route, the signal strength, and traffic load are used as Choosing indicators, this scheme can only reach the local optimum, but not the global optimum. When the number of communication hops increases, there may be a contradiction between the local optimal and the shortest hops. Based on the LTA-OLSR protocol, the LCO-OLSR protocol broadcasts local information to nodes on the entire network through TC messages, achieving a balance between the global optimal and the shortest number of hops.

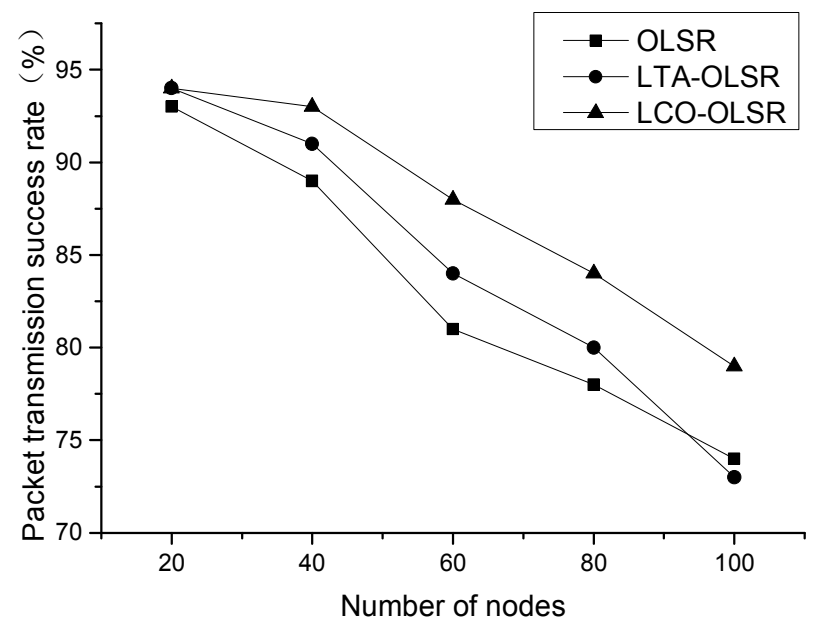

Figure 8. Comparison of success rate of outsourcing.

4) Analysis of node moving speed on success rate of packet sending

In the scenario of 40 nodes, different maximum movement speeds, and corresponding success rates of the packet sending are counted. Figure 8 shows that as the node movement speed increases, the LCO-OLSR protocol is gradually equal to the OLSR protocol in the index of packet sending success rate. The reason is that as the node moving speed increases, the number of stable links in the network will decrease. When $\mathrm{V}_{\max }=33 \mathrm{~m} / \mathrm{s}$ is obtained from formula 2 , there will be no stable links in the network, and the LCO-OLSR protocol will degenerate into the OLSR protocol.

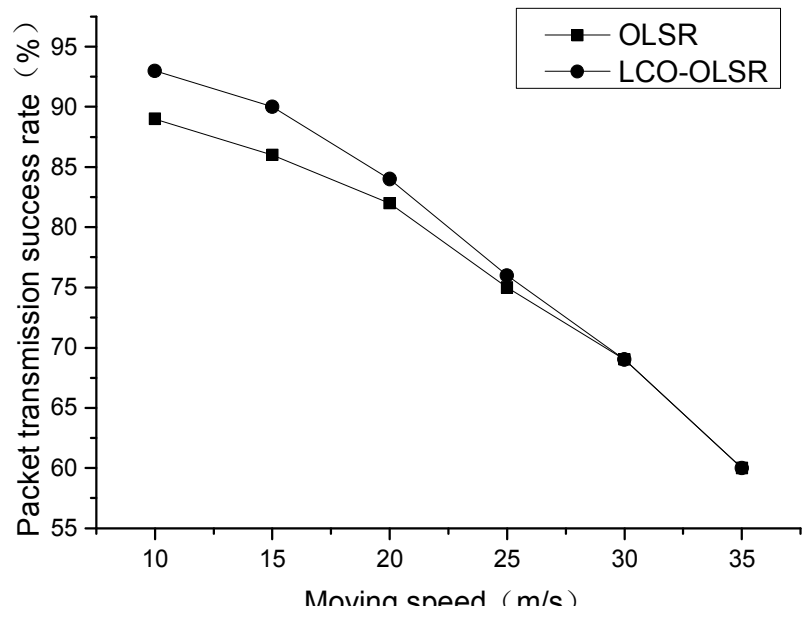

Figure 9. The effect of speed on the success rate of sending packets.

\section{Conclusion}

This paper proposes an OLSR protocol for optimizing 
routing with low control overhead for UAV ad hoc networks. By using incremental HELLO message mechanism and routing optimization mechanism, the problems of high control overhead, high channel occupancy and multi-hop link communication interruption are improved. Theoretical analysis and simulation results show that the LCO-OLSR protocol can clearly improve control overhead, end-to-end delay, and packet delivery success rate in low-dynamic scenarios. However, the experimental simulation results show that the performance of the protocol gradually degrades to the OLSR protocol under high dynamic scenarios. In future work, we will study in depth to improve the performance of the OLSR protocol in highly dynamic scenarios.

\section{References}

[1] Z. Yi, W. Yuwen, L. Zhenzhen, D. Li, J. Yu, and Z. Hong, "A Mobility and Load aware OLSR routing protocol for UAV mobile ad-hoc networks," in Proc. 2014 International Conference on Information and Communications Technologies (ICT 2014), Nanjing, China, May. 2014, pp. 1-7.

[2] S. Y. Dong, "Optimization of OLSR routing protocol in UAV ad HOC network," in Proc. 2016 13th International Computer Conference on Wavelet Active Media Technology and Information Processing (ICCWAMTIP), Chengdu, China, Dec. 2016, pp. 90-94.

[3] V. Sanchez-Aguero, F. Valera, B. Nogales, L. F. Gonzalez, and I Vidal, "VENUE: Virtualized Environment for Multi-UAV Network Emulation," IEEE Access, vol. 7, pp. 154659-154671, 2019.

[4] K. Singh and V. Anil Kumar, "Experimental analysis of AODV, DSDV and OLSR routing protocol for flying ad hoc networks (FANETs)," in Proc. 2015 IEEE International Conference on Electrical, Computer and Communication Technologies (ICECCT), Coimbatore, India, Mar. 2015, pp. 1-4.

[5] J. Hao, G. Duan, B. Zhang, and C. Li, "An energy-efficient on-demand multicast routing protocol for wireless ad hoc and sensor networks," in Proc. 2013 IEEE Global Communications Conference (GLOBECOM), Atlanta, GA, USA, Dec. 2013, pp. 4650-4655.

[6] Y. Jiang, Z. Mi, H. Wang, X. Wang, and N. Zhao, "The experiment and performance analysis of multi-node UAV ad hoc network based on swarm tactics," in Proc. 2018 10th International Conference on Wireless Communications and Signal Processing (WCSP), Hangzhou, China, Oct. 2018, pp. $1-6$.

[7] A. V. Leonov and G. A. Litvinov, "Applying AODV and OLSR routing protocols to air-to-air scenario in flying ad hoc networks formed by mini-UAVs," in Proc. 2018 Systems of
Signals Generating and Processing in the Field of on Board Communications, Moscow, Russia, Mar. 2018, pp. 1-10.

[8] Y. Wang, Z. Wei, X. Chen, H. Wu, and Z. Feng, "Demo: UAV assisted adaptive aerial internet," in Proc. 2018 IEEE/CIC International Conference on Communications in China (ICCC), Beijing, China, Aug. 2018, pp. 732-733.

[9] A. Waheed, A. Wahid, and M. A. Shah, "LAOD: Link aware on demand routing in flying Ad-Hoc networks," in Proc. 2019 IEEE International Conference on Communications Workshops (ICC Workshops), Shanghai, China, May 2019, pp. $1-5$.

[10] F. Wang, Z. Chen, J. Zhang, C. Zhou, and W. Yue, "Greedy forwarding and limited flooding based routing protocol for UAV flying Ad-Hoc networks," in Proc. 2019 IEEE 9th International Conference on Electronics Information and Emergency Communication (ICEIEC), Beijing, China, July. 2019, pp. 1-4.

[11] S. N. Pari and D. Gangadaran, "A reliable prognostic communication routing for flying ad hoc networks," in Proc. 2018 2nd International Conference on Trends in Electronics and Informatics (ICOEI), Tirunelveli, India, May. 2018, pp. 33-38.

[12] M. Song, J. Liu, and S. Yang, "A mobility prediction and delay prediction routing protocol for UAV networks," in Proc. 2018 10th International Conference on Wireless Communications and Signal Processing (WCSP), Hangzhou, China, Oct. 2018, pp. 1-6.

[13] A. I. Alshbatat and L. Dong, "Cross layer design for mobile Ad-Hoc unmanned aerial vehicle communication networks," in Proc. 2010 International Conference on Networking, Sensing and Control (ICNSC), Chicago, IL, USA, Apr. 2010, pp. 331-336.

[14] C. Pu, "Link-Quality and traffic-load aware routing for UAV Ad Hoc networks," in Proc. 2018 IEEE 4th International Conference on Collaboration and Internet Computing (CIC), Philadelphia, PA, USA, Oct. 2018, pp. 71-79.

[15] H. R. Hussen, S. Choi, J. Park, and J. Kim, "Performance analysis of MANET routing protocols for UAV communications," in Proc. 2018 Tenth International Conference on Ubiquitous and Future Networks (ICUFN), Prague, Czech Republic, July. 2018, pp. 70-72.

[16] P. Xie, "An enhanced OLSR routing protocol based on node link expiration time and residual energy in ocean FANETS," in Proc. 2018 24th Asia-Pacific Conference on Communications (APCC), Ningbo, China, Nov. 2018, pp. 598-603.

[17] L. Li, X. Yixiang, H. Xiaoguang, D. Haibin, Z. Hanyu, C. Jun, and L. Jin, "A new rechargeable WSNs based Multi-UAVs network and topology control algorithm," in Proc. 2015 IEEE 10th Conference on Industrial Electronics and Applications (ICIEA), Auckland, New Zealand, June. 2015, pp. 507-512. 